\title{
Nanosphere lithography for optical fiber tip nanoprobes
}

\author{
Marco Pisco ${ }^{1, \star}$, Francesco Galeotti ${ }^{2, \star}$, Giuseppe Quero ${ }^{1}$, Giorgio Grisci ${ }^{2}$, Alberto Micco ${ }^{1}$, Lucia V Mercaldo ${ }^{3}$, \\ Paola Delli Veneri ${ }^{3}$, Antonello Cutolo ${ }^{1}$ and Andrea Cusano ${ }^{1}$
}

This paper reports a simple and economical method for the fabrication of nanopatterned optical fiber nanotips. The proposed patterning approach relies on the use of the nanosphere lithography of the optical fiber end facet. Polystyrene (PS) nanospheres are initially self-assembled in a hexagonal array on the surface of water. The created pattern is then transferred onto an optical fiber tip (OFT). The PS monolayer colloidal crystal on the OFT is the basic building block that is used to obtain different periodic structures by applying further treatment to the fiber, such as metal coating, nanosphere size reduction and sphere removal. Ordered dielectric and metallo-dielectric sphere arrays, metallic nanoisland arrays and hole-patterned metallic films with feature sizes down to the submicron scale are achievable using this approach. Furthermore, the sizes and shapes of these periodic structures can be tailored by altering the fabrication conditions. The results indicate that the proposed self-assembly approach is a valuable route for the development of highly repeatable metallo-dielectric periodic patterns on OFTs with a high degree of order and low fabrication cost. The method can be easily extended to simultaneously produce multiple fibers, opening a new route to the development of fiber-optic nanoprobes. Finally, we demonstrate the effective application of the patterned OFTs as surface-enhanced Raman spectroscopy nanoprobes.

Light: Science \& Applications (2017) 6, e16229; doi:10.1038/lsa.2016.229; published online 19 May 2017

Keywords: lab-on-fiber; nanosphere lithography; SERS probes

\section{INTRODUCTION}

The integration of microscale and nanoscale functional materials and structures synergistically combined in a single platform based on optical fiber technology creates unrivalled opportunities for the realization of advanced optical fiber devices. Specifically, the fabrication of nanostructures on optical fiber tips (OFTs) facilitates extraordinary light trapping and manipulation at the nanoscale, enabling the realization of compact optical systems and advanced OFT devices with smart characteristics that are suitable for various sensing purposes.

Optical fibers are well suited for sensing applications due to the intrinsic advantages associated with their use, such as remote operation capability, low intrusiveness and stability in harsh environments. The periodic patterns of the nanoscale structures can operate as miniaturized optical components and implement optical transduction mechanisms on a tiny spatial scale. The integration of periodic nanoscale structures onto OFTs enables the exploitation of cutting edge physical mechanisms arising from operating at subwavelength scale, as is the case of the excitation of photonic and plasmonic resonances, to provide ready-to-use substrates for surface-enhanced Raman spectroscopy (SERS).

Recently, the significant growth in the field of nanotechnology has made it possible to fabricate nanopatterns directly on OFTs. These efforts, which collectively focus on the fabrication challenges related to the use of optical fibers as an exclusive substrate for micro- and nanotechnologies ${ }^{1}$, are often termed 'lab-on-fiber' technologies ${ }^{2,3}$. In this new technological paradigm, a novel class of multifunctional optical fiber components and systems is envisioned, fusing nanotechnology, materials science, and optics.

The processes that are commonly used to fabricate micro- and nanopatterns on OFTs (that is, electron beam ${ }^{4-6}$ and focusing ion beam lithography ${ }^{7-10}$ ) can precisely control the dimensions of the nanostructures. However, these methods require expensive instrumentation, and the related fabrication process is inherently time consuming.

In contrast, processes based on self-assembly represent an easier, quicker and cheaper option than nanolithography for the fabrication of regular micro- and nanopatterns ${ }^{11}$. In addition, self-assembly methods can easily be scaled from a single fiber to a bundle of optical fibers, allowing the simultaneous creation of an ordered pattern on multiple fibers and paving the way for the mass production of OFT devices.

Some of the earliest attempts to use self-assembly techniques to decorate OFTs were motivated by their use as SERS probes and relied on surface roughness rather than on the presence of a regular pattern $^{1,12}$. For example, metal nanorods and nanoislands have been deposited on OFTs by evaporation, but in both cases, the degree of order achieved was quite low ${ }^{13,14}$. Similarly, Stoddart et al. ${ }^{15}$ evaluated

${ }^{1}$ Optoelectronic Division_Engineering Department, University of Sannio, 82100 Benevento, Italy; ${ }^{2}$ National Research Council, Institute for Macromolecular Studies (ISMAC-CNR), 20133 Milano, Italy and ${ }^{3}$ ENEA, Portici Research Center, 80055 Portici, Italy

*These authors contributed equally to this work.

Correspondence: A Cusano, Email: a.cusano@unisannio.it

Received 14 March 2016; revised 6 September 2016; accepted 6 September 2016; accepted article preview online 8 September 2016 
the use of a simple 'dip and dry' method for producing periodic nanoparticle arrays on OFTs, which resulted in a combination of mono- and multilayer zones and inhomogeneous fiber coverage. Yap et al. arranged gold nanoparticle clusters on OFTs using an electrostatic self-assembly approach guided by a block copolymer template. Although this method yielded ordered clusters on a planar substrate, an attempt to reproduce the process on fibers resulted in close-packed but unordered gold clusters because of the insufficient ordering of the polymer template ${ }^{16}$. In summary, the fabrication of periodic nanostructures on OFTs via self-organization remains challenging.

Our group previously reported a self-assembly method based on breath figure arrays for the fabrication of ordered nanostructures on $\mathrm{OFTs}^{17}$. In this approach, under controlled conditions, the evaporation of a solvent from a polymer solution triggers the condensation of micrometric water droplets that self-assemble into a hexagonal lattice. The water then evaporates, and the droplet lattice leaves an ordered microporous pattern on the polymeric film. Using the optical fiber end facet as a substrate, we obtained sensing nanoprobes with definite metallo-dielectric porous surfaces.

However, major limitations of that approach were related to the size of the water droplets. It was difficult to obtain holes with a diameter of less than one micron, and the aspect ratio (the ratio between the hole radius and the pattern period) could not be easily controlled.

Thus, to overcome the issues of the previous method and to expand the set of self-assembly procedures for the realization of ordered nanostructures on OFTs, a pioneer study was conducted by our group to investigate the self-assembly of colloidal spheres ${ }^{18}$ on OFTs. The key aspect of the proposed method relies on the formation of monolayer colloidal crystals (MCCs) of commercially available nanospheres of polystyrene (PS) at the interface between air and water (AWI ${ }^{19-26}$. In our procedure, the MCC floating at the AWI is captured on the facet of an OFT. By successively applying additional fabrication steps based on thermal evaporation, plasma etching and sonication, different periodic structures are obtained. The preliminary investigation demonstrated the ability of the approach to produce ordered patterns of nanospheres on OFTs ${ }^{18}$.

Starting with these promising results, here we study the possibility of adapting the nanosphere lithography ${ }^{27}$ to fabricate repeatable patterns on $\mathrm{OFTs}^{28}$. A careful experimental study has been conducted to optimize the operating conditions for each fabrication step. Focused ion beam microscopy (FIBM) and atomic force microscopy (AFM) have been exploited to assess the morphological structure of the patterns directly on an OFT. This study represents a thorough experimental analysis devoted to the identification and assessment of a simple and economical self-assembly methodology to create nanotemplates on an OFT. A careful analysis was also conducted to investigate the ability of the proposed procedure to realize repeatable patterns on an OFT, which is a key requirement for new multifunctional photonics nanoprobes.

Finally, we demonstrate how the optimized process provides a valuable tool for the realization of fiber-optic SERS probes that outperform the prototypes demonstrated in our pioneering work ${ }^{18}$.

\section{MATERIALS AND METHODS}

\section{Instruments}

Plasma etching was performed on a BOC Edwards Scancoat Six Sputter Coater (Crawley, UK) at $\sim 50 \mathrm{~W}\left(3 \times 10^{-1} \mathrm{mbar}\right)$. An FEI Quanta 3D FEG dual beam instrument (FEI, Hillsboro, OR, USA), comprising a highly focused ion beam (FIB) and a scanning electron microscope (SEM), was used to analyze the morphology of the fabricated samples. AFM analyses were conducted with a NT-MDT
NTEGRA instrument (Moscow, Russia) operating in semicontact mode.

\section{Fabrication procedure}

The concentration of commercial PS nanosphere suspensions (2.5\% wt in water, Polysciences Europe GmbH, Hirschberg an der Bergstrasse, Germany) was adjusted by precipitation in a microultracentrifuge and resuspension in different volumes of 1:1 ethanol/ water according to the sphere diameter. To optimize the MCC homogeneity, the final weight concentrations were set as follows: $200 \mathrm{~nm}$ microspheres at $8 \%, 350$ and $500 \mathrm{~nm}$ at $10 \%, 750 \mathrm{~nm}$ at $15 \%$, and $1000 \mathrm{~nm}$ at $6 \%$. The hydroalcoholic colloidal solutions were sonicated for 2 min to break up the aggregates and were then slowly deposited on the surface of water using a $50 \mu \mathrm{l}$ micro-syringe through a Si conduit plate (Figure 1). The conduit plate helped to mitigate sharp compression and to prevent the formation of nanosphere aggregates resulting from van der Waals forces. The plate was obtained by cutting a $6 \times 20 \mathrm{~mm}^{2}$ piece from a standard Si wafer that was exposed to air plasma for $300 \mathrm{~s}$ to make its surface more hydrophilic and then stored in a 1:1 ethanol/water solution until use. During the nanosphere deposition, one edge of the conduit plate was kept immersed $(\approx 2 \mathrm{~mm})$ almost perpendicularly in a glass beaker filled with ultrapure water inside a confinement ring (a standard fluoroelastomer o-ring), which confined the MCC in a restricted area of the water surface. Because of the hydrophobic nature of its surface, the confinement ring induced a convex meniscus in the water, preventing the MCC islands from migrating and sticking to the walls of the beaker. The nanosphere suspension was released on the conduit plate at $\sim 3 \mu \mathrm{min}^{-1}$. As soon as it reached the end of the plate, the suspension rapidly spread on the surface of the water, letting the nanospheres self-assemble in a close-packed array (CPA) and forming a floating MCC at the AWI. A 20-30 $\mu$ l volume of nanosphere suspension was generally sufficient to fill a $4 \mathrm{~cm}$ diameter confinement ring with MCC, as shown in Figure 1, with a total deposition time of $\sim$ 15-20 min. The precise volume and, consequently, deposition time, depended on the sphere diameter and on the floating yield. After the MCC was formed, it was transferred onto an OFT by dipping the fiber using tweezers into the same deposition beaker and gently lifting it toward the MCC island floating on the surface. The patterned OFT was then dried with a flow of nitrogen. The CPA structure of the OFT nanoprobe was obtained by covering the fiber with a thin gold coating $(30-40 \mathrm{~nm})$. Selective sphere removal was performed by immersing the patterned OFT in distilled water and sonicating in an ultrasound bath for a time ranging from $30 \mathrm{~s}$ for the $1-\mu \mathrm{m}$ spheres to $5 \mathrm{~min}$ for the 200-nm spheres. This process formed the CPA-sphere removal (SR). To reduce the size of the reliefs, the OFT covered with PS nanospheres (before metallization) was exposed to air plasma for an amount of time that depended on the initial sphere size and the desired size reduction. Regardless of the total etching time, the plasma was switched off after $200 \mathrm{~s}$ of exposure, and the chamber was allowed to cool for a few minutes before starting the subsequent $200 \mathrm{~s}$ of treatment to prevent the temperature increase caused by etching from melting the PS. The metallization of these plasma-etched OFTs produced sparse array (SA) nanoprobes. The ultrasound bath treatment of the metallized SA patterns produced the SA-SR configuration.

\section{Spectral characterization}

The transmittance spectrum of the CPA sample was obtained by illuminating it with a supercontinuum white light source with a filter for the visible spectrum (SuperK Compact, NKT Photonics, Birkerød, Denmark) and collecting the transmitted optical power directly with 


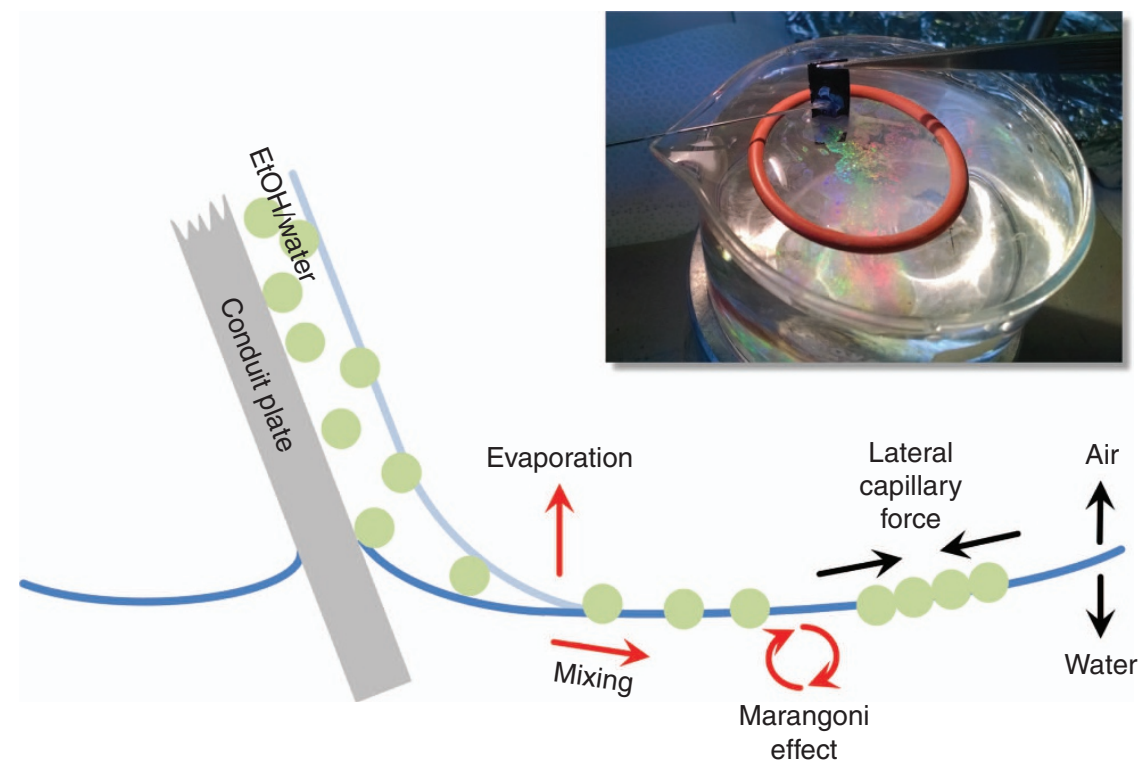

Figure 1 Schematic of the nanosphere assembly process and photograph of MCC islands (green blotches) floating on water.

an optical spectrum analyzer (Ando Electric, AQ6317C, Tokyo, Japan). The power transmitted through the sample was then normalized by the transmission of the same light source through an optical fiber patch cord.

\section{SERS experiments}

SERS measurements were performed with a commercial spectroscopic Raman system (Renishaw InVia, Gloucestershire, UK) with a 785-nm laser source. The excitation light was focused on the sample through a $50 \times$ objective lens (without going through the optical fiber), which was also used to collect the resulting Raman signals (see the schematics in the Supplementary Information, Supplementary Fig. S1). Before each Raman measurement, the system was calibrated using a silicon standard at $520 \mathrm{~cm}^{-1}$.

OFT samples were soaked in $1-\mu \mathrm{M}$ crystal violet $(\mathrm{CV})$ for $3 \mathrm{~h}$ immediately before the SERS measurements. The SERS spectra acquisitions were performed on five random locations with an exposure time of $10 \mathrm{~s}$. The locations were in the cladding area to guarantee proper spatial separation. The SERS spectrum at the geometric center of the fiber facet (fiber core) was also acquired. The repeatability of the measurements at the same location was verified. The baseline was subtracted from the spectra to eliminate the unwanted background and to facilitate data analysis.

\section{RESULTS AND DISCUSSION}

\section{Nanoprobe fabrication}

The proposed strategy for the development of multifunctional optical fiber nanoprobes requires the self-assembly of PS nanospheres with a predefined diameter at the AWI and the successive transfer of the resulting MCC onto an OFT. Compared with other nanosphere assembly approaches, such as capillary ${ }^{29}$, sedimentation ${ }^{30}$ and spincoating deposition $^{31}$, assembly at the AWI has the following benefits: (i) water is an ideal deposition substrate because its surface is easy to clean by refreshing; (ii) MCC assembly takes only a few seconds; and (iii) the assembly requires minimal quantities of nanoparticles.

The idea of assembly at the AWI is adopted from the LangmuirBlodgett procedure. In that technique, a solution of organic molecules dissolved in a non-polar solvent, such as dichloromethane or hexane, is spread over the surface of water ${ }^{32}$. Similarly, in the AWI assembly approach, the nanospheres are deposited on water from a water/ alcohol suspension. Ethanol is added to decrease the surface tension of the aqueous colloidal solution, thereby enhancing the spread of nanospheres on the water.

The assembly process is shown in Figure 1. As soon as the hydroalcoholic suspension of PS nanospheres is released onto the water surface, it rapidly spreads, creating a momentary alcohol-water bilayer. Consequently, the PS spheres are pushed by the spreading and evaporation of alcohol and are distributed over the water surface. The driving force of this process is related to the Marangoni effect; as the hydroalcoholic PS colloidal solution contacts the water surface, strong Marangoni forces are formed, pushing the colloidal particles to disperse rapidly outward from regions with low surface tension until they cover the whole surface of the water bath. Within a few seconds, some of the ethanol evaporates, and the remainder mixes with the water. Thus, most of the nanospheres are left floating on the water surface and start to self-assemble through capillary forces and collective motion. To obtain a high-quality assembly, it is important that only a small fraction of the nanospheres sink in the water and fail to participate in MCC formation. Therefore, an appropriate concentration for each nanosphere suspension, as reported in the previous section, is crucial to the formation of the MCC at the AWI.

After the MCCs form (shown in the photograph in Figure 1), they are transferred onto an OFT. During this step, the main difficulty is the size of the collecting substrate, that is, the facet area of a standard monomodal fiber $(125 \mu \mathrm{m}$ in diameter $)$ is too small to collect a portion of the MCC without damaging it. To solve this problem, we use an OFT embedded in a ceramic ferrule (similar to those used in standard fiber connectors), which gives the OFT a wider exposed surface $(2.5 \mathrm{~mm}$ of diameter) than the pristine fiber, enhancing the ease of handling in all steps of the patterning process. Hence, we can successfully collect the assembled spheres on the modified OFT by dipping the fiber in the water reservoir used to prepare the assembly and then gently lifting it toward the MCC island floating on the surface. When a portion of the MCC comes into contact with the 
optical fiber face, it sticks because of the hydrophobicity of the PS spheres; thus, the MCC can be dried under a gentle nitrogen flow without breakage. When the water evaporates, the PS spheres assemble together in a close-packed hexagonal pattern through capillary motion and become tightly attached to the fiber by electrostatic and van der Waals attractive forces.

Although this operation was performed manually by dipping and lifting a single OFT with tweezers, it could be easily scaled-up by simultaneously dipping a large number of optical fibers. Because very large MCCs can theoretically be fabricated by enlarging the surface area of the deionized water, in principle, the number of fibers that can be processed simultaneously is not limited; thus, this process is well suited for industrial production.

To manufacture different structural motifs and to explore various possibilities for obtaining periodic patterns on the OFTs, we manipulated the MCC-decorated fibers, as shown in Figure 2. After collecting an MCC on the fiber, further treatment (gold coating, plasma etching and sonication) was applied to obtain structures with different features.

Specifically, by depositing a thin metallic layer (that is, gold) onto the fiber/MCC assembly by thermal evaporation, we obtained a metallo-dielectric crystal (which resulted in the simplest case of $\mathrm{CPA}$ ). After this treatment, the resulting OFT shows a bright iridescence caused by the Bragg reflection of ambient light through the OFT patterns, as shown in the digital photograph in Figure 2. The immersion of the OFT in water and successive ultrasound treatment leads to SR, and an ordered pattern of metallic triangles shaped as a six-point star (CPA-SR) is left on the fiber. Alternatively, exposing the fibers covered with nanospheres (before metallization) to oxygen plasma conveniently decreases sphere diameter. This treatment makes it possible to tune the size of the spheres assembled on the OFT without changing the patterning period. A metallo-dielectric SA of reliefs is obtained by metallizing these fibers. Then, after metal coating, the plasma-etched nanospheres can be polished off the OFT by immersion in an ultrasound bath, yielding a porous gold mask (SA-SR).

To investigate the feasibility of this approach for fabricating metallo-dielectric periodic patterns on optical fibers, we used PS spheres with five diameters: 200, 350, 500, 750 and $1000 \mathrm{~nm}$. This size range was selected so that the resulting periodic arrays featured subwavelength dimensions, which are interesting for the development optical fiber sensors operating in ultraviolet-visible and infrared wavelengths.

For each sphere diameter, we fabricated an MCC assembly on an OFT. We obtained a CPA of metallo-dielectric spheres by metallization with a thermally evaporated $30-40 \mathrm{~nm}$ gold layer. As shown in Figure 3, this procedure decorated the OFT with highly ordered MCCs, regardless of the sphere size, except for those spheres with a diameter of $200 \mathrm{~nm}$, which resulted in some irregularities. The AFM profiles (insets in Figure 3) confirm that the surface morphology of the fiber facet was modified with well-defined periodic patterns because of the presence of gold-coated nanospheres.

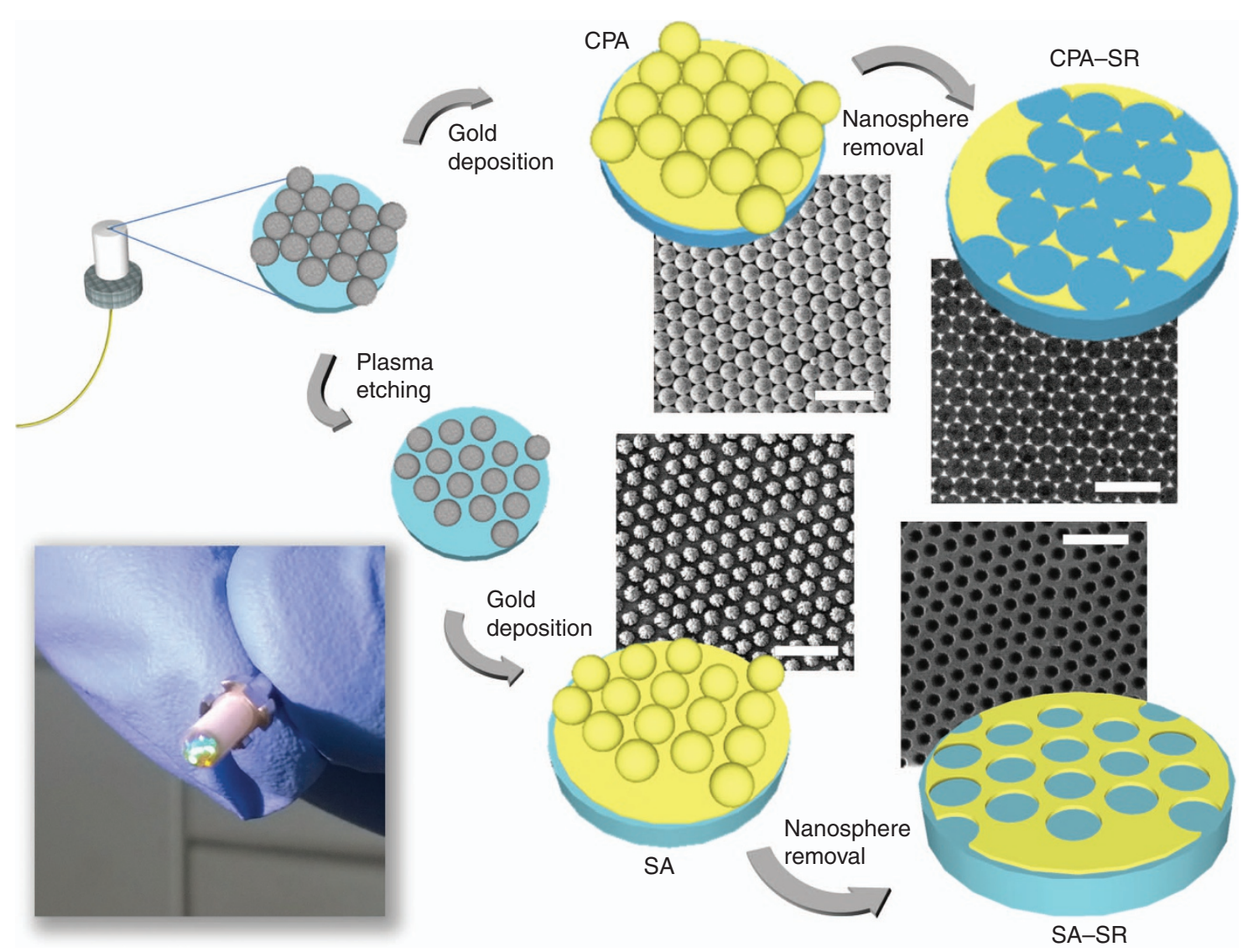

Figure 2 Schematic representing the fabrication procedure for creating different fiber tip nanoprobes and SEM images (scale bar $=3 \mu \mathrm{m}$ ) of real samples obtained using 1- $\mu \mathrm{m}$ nanospheres, corresponding to each geometric feature: CPA, SA and SR. A digital picture of a fiber tip showing an iridescent nanopattern is also shown. 
a

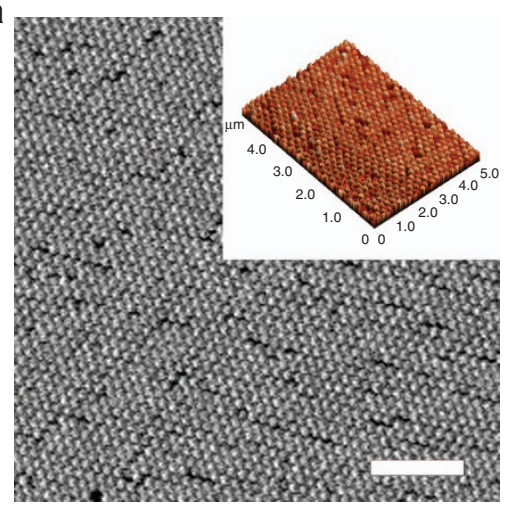

d

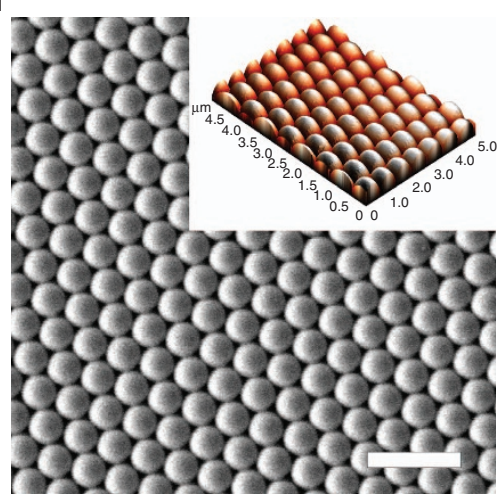

b

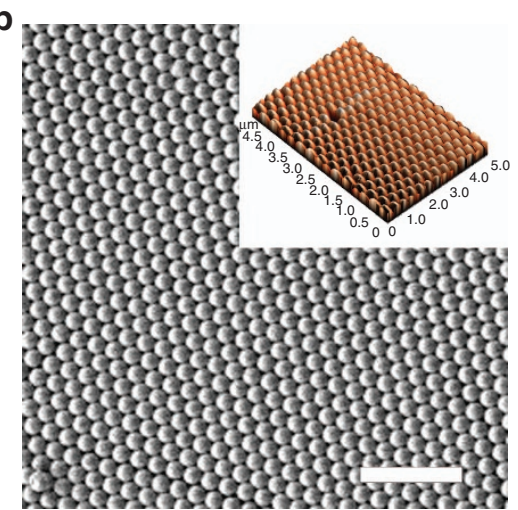

e

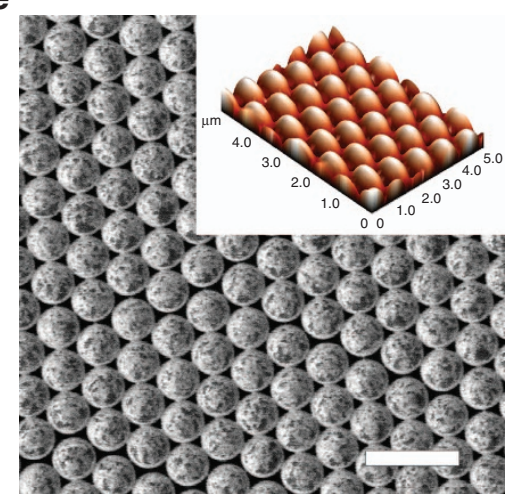

C

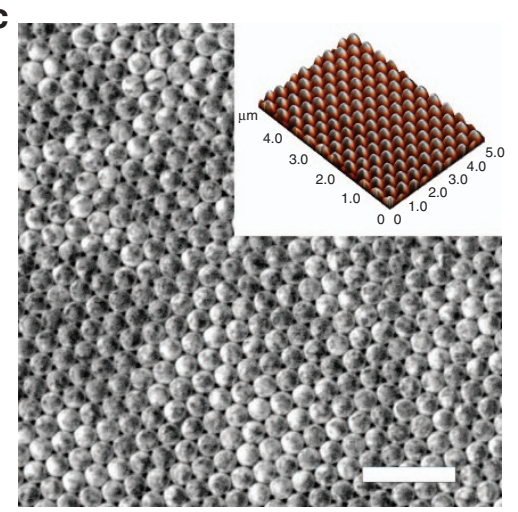

f

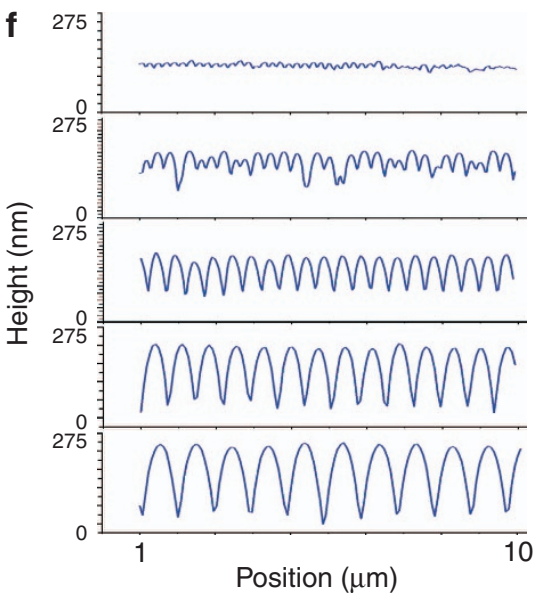

Figure 3 (a-e) FIBM and AFM (inset) images of OFTs patterned with CPA metallo-dielectric nanospheres with diameters of 200, 350, 500, 750 and $1000 \mathrm{~nm}$. Scale bars $=2 \mu \mathrm{m}$. (f) AFM section profiles of the same samples from the top $(200 \mathrm{~nm})$ to bottom $(1000 \mathrm{~nm})$. Figure reprinted with permission from Pisco et al. ${ }^{18}$.

To selectively remove the spheres while leaving the gold pattern on the fiber surface, the patterned OFT was immersed in distilled water and sonicated in an ultrasound bath.

Figure 4 shows the periodic patterns obtained after executing the steps to achieve CPA-SR for the various sphere sizes. The triangular gold structures of the samples are not interconnected; their spatial arrangements depend on the dimensions of the nanospheres used to prepare the MCCs, whereas their heights depend on the thickness of the gold layer that was thermally evaporated onto the spheres. For example, for the 200-, 500- and 1000-nm spheres, the average measured triangle sides were 80,150 and $280 \mathrm{~nm}$, respectively, and their heights after depositing a 30-nm layer of gold were $\sim 30 \mathrm{~nm}$. Consequently, the aspect ratio of the three-dimensional periodic nanostructures also varies considerably, with the smallest period corresponding to the highest aspect ratio $(\approx 0.3)$.

Another configuration (SA) was obtained by applying plasma etching pretreatment to the MCC on the fiber tip before metallization. The period of the SA pattern is determined by the pristine nanosphere diameter, whereas the final size of the reliefs depends on the etching time. To decrease the size of the nanospheres, the OFT can be exposed to air plasma.

Figure 5 presents the FIBM images of the sample obtained by exposing fibers covered with 1000-nm MCC to $600 \mathrm{~s}$ of air plasma etching and then evaporating the gold layer, as for the previous samples. As indicated by the top views of the fiber in panels $a$ and $b$, plasma etching considerably reduced the diameter of the spheres, producing a wide gap surrounding the metallo-dielectric reliefs. The period of the pristine MCC was maintained in this periodic structure, resulting in an array with a period of $1000 \mathrm{~nm}$, whereas both the height and the diameter of the reliefs decreased to $\sim 600 \mathrm{~nm}$.

The tilted FIBM view (at an angle of $52^{\circ}$ ) and its zoomed detail (panels $\mathrm{c}$ and d) confirm that the plasma etching of the PS spheres is anisotropic, as previously reported by Akinoglu et al. ${ }^{33}$. This leads to a considerable change in the relief shape, that is, from a sphere to a spheroid particle with a biconvex form, as shown in the sketch. The degree of anisotropy becomes increasingly evident as the etching time increases. The lateral surfaces of these biconvex reliefs have a groovy nanostructure, which may contribute the optical response of these types of periodic patterns.

The last type of structure is obtained starting from the SA pattern. As shown in Figure 6, treating the SA fibers in an ultrasound bath resulted in complete removal of the particles, leaving a hexagonally ordered, nanoporous gold array on the fiber surface, as expected for the SA-SR fabrication route. Samples of SA-patterned fibers obtained from 500- and 1000-nm spheres are in panels a and e, respectively, and panels $b$ and $f$ present the corresponding SA-SR samples. The AFM section profiles of both samples reveal that the periods of the pristine MCCs (500 and $1000 \mathrm{~nm}$ ) are maintained and that the diameters of the holes are $\sim 250$ and $500 \mathrm{~nm}$, respectively, as determined based on the gold layer thickness $(\approx 40 \mathrm{~nm}$, Figure $6 \mathrm{c}$ 

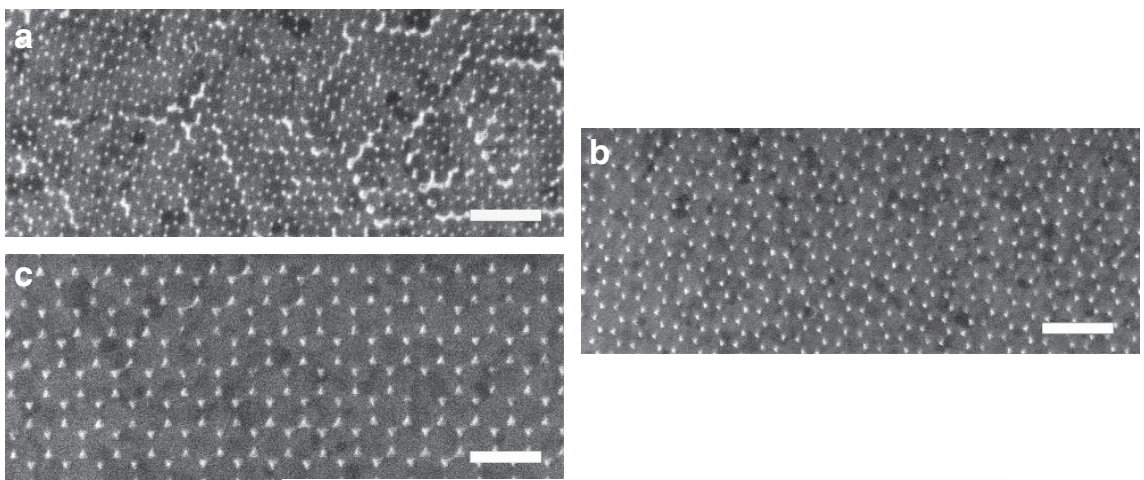

d
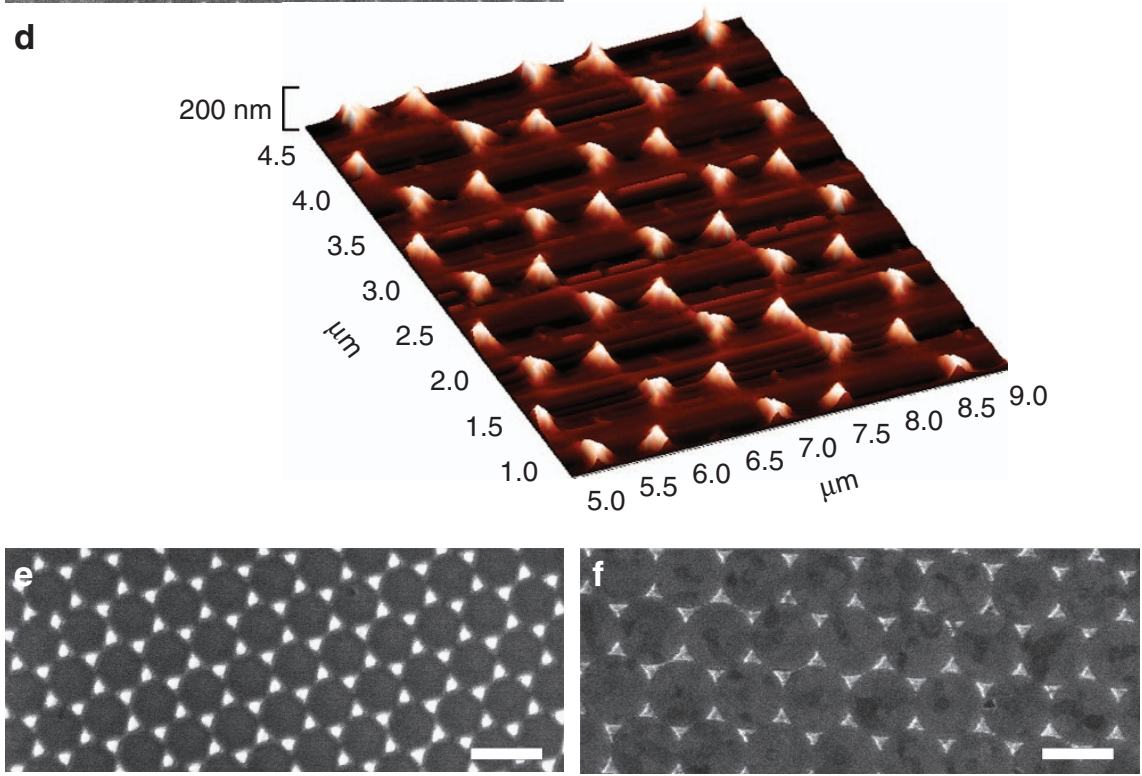

Figure 4 FIBM micrographs (a-c, e, f) and AFM three-dimensional image (d) of fiber tips patterned with CPA-SR gold structures. The periods are 200 (a), 350 (b), 500 (c), 750 (e) and $1000 \mathrm{~nm}(\mathbf{d}, \mathbf{f})$. The FIBM scale bars are $1 \mu \mathrm{m}$.

and $6 \mathrm{~d}, 6 \mathrm{~g}$ and $6 \mathrm{~h})$. In summary, we systematically investigated the possibility of adopting and modifying the nanosphere lithography of optical fibers, that is, the fabrication substrate, and obtained different structures on OFTs. All these periodic structures have already been demonstrated using planar substrates and can generally be used in various operational conditions ${ }^{18}$. Nevertheless, we demonstrated for the first time that this level of precision can also be achieved on OFTs using a self-assembly approach and simple instrumentation that is available in most laboratories.

Even though the nanoprobes feature regular and well-ordered patterns, the method still suffers from the formation of defects. To provide a picture of the process yield, we analyzed $10 \mathrm{CPA}$ samples fabricated with 1000-nm diameter PS nanospheres. Among them, three were discarded after an initial visual inspection by AFM because they presented several agglomerates of spheres, breaking the regularity of the pattern (see Supplementary Fig. S2a). The retained samples exhibit regular structures on the fiber tip with a certain degree of defects remaining, leading to poor repeatability (AFM images of representative samples are shown in Supplementary Fig. S2b). The presence of defects indicates that the process requires further improvement to ensure sufficient repeatability, especially as the described procedure involves several manual steps, which are considered the main source of uncertainty.
Process optimization and reproducibility assessment

To improve our fabrication method aimed to integrate self-assembled nanopatterns onto OFTs, it is necessary to avoid manual steps and to introduce highly controlled stages to ensure a high process yield rate and to improve the repeatability. To discriminate between human errors and process faults, we fixed the main sources of uncertainty in the fabrication process, which are mainly related to the nanosphere deployment onto the water surface and to the final transfer step. In this experimental stage, the deposition rate was controlled by using a syringe pump, and the fiber lifting was performed using a mechanical lever.

To verify the improvement with respect to the previous manual approach, we fabricated ten CPA samples with a diameter of $1000 \mathrm{~nm}$ and analyzed the resulting morphology by AFM. The fabrication of the CPA samples involves the formation of the MCC at the AWI and its transfer onto a fiber tip, so it is representative of the most critical steps of the proposed fabrication route.

In addition, to investigate the capability of the proposed procedure to produce homogeneous and repeatable patterns on the optical fiber, we selected SA as benchmark, which compared with the CPAs samples requires an additional fabrication step (that is, sphere reduction) that could potentially affect the pattern regularity. In this way, by analyzing the morphology of the SA samples, we could analyze the repeatability of all critical fabrication steps. Thermal evaporation and sphere 

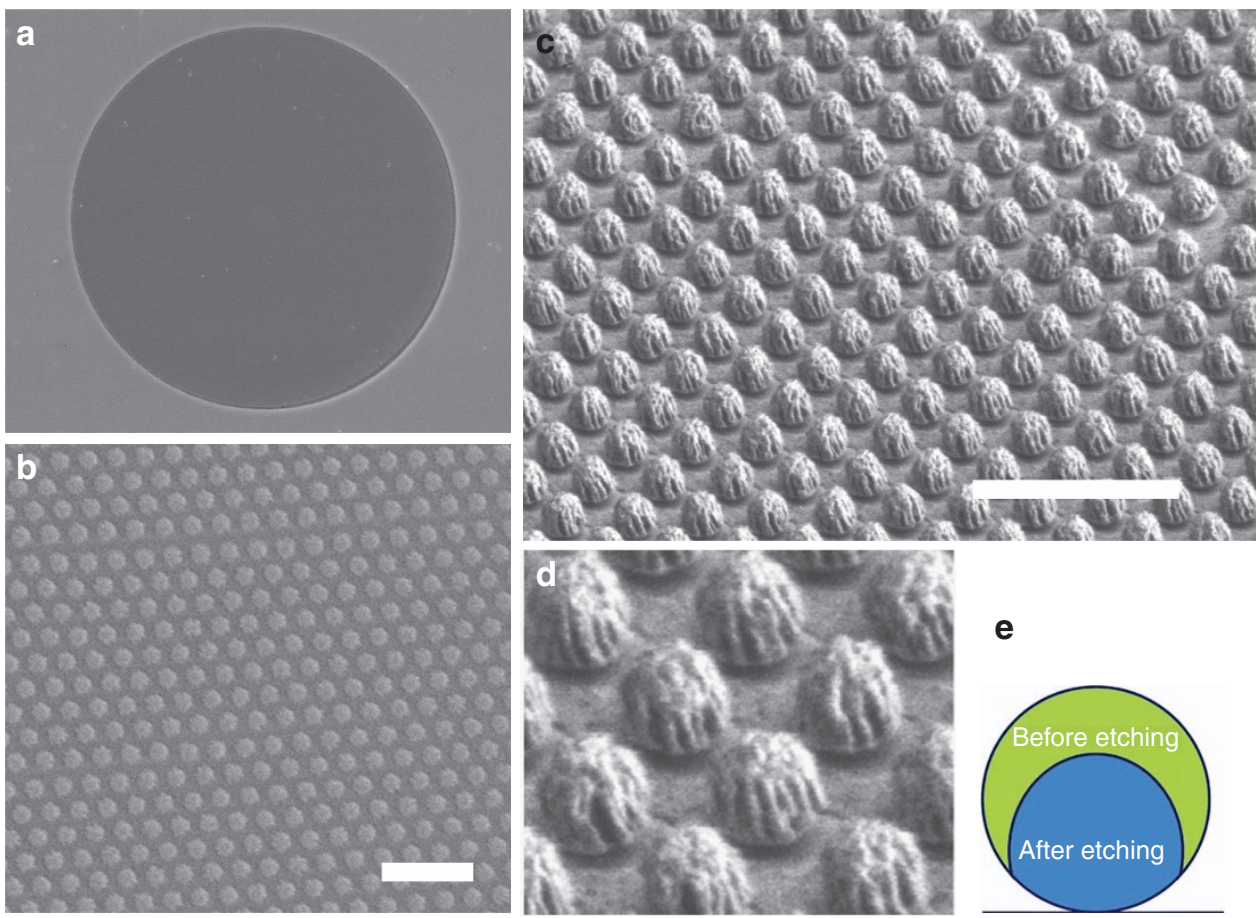

Figure 5 Top (a, b) and tilted (c, d) FIBM views showing the PS spheres after size reduction (SA patterns) and a sketch of the anisotropic etching of a spherical particle (e). Scale bars $=3 \mu \mathrm{m}$.

removal via sonication are effective fabrication processes and are not considered critical steps in the overall fabrication route. Finally, spectral analysis provides an understanding of the real impact of the observed morphological differences on the resulting device for both CPA and SA samples.

According to this approach, we repeated the assembly at the AWI using the 1000-nm spheres 10 times and then transferred a portion of the obtained MCC onto 10 fibers to obtain, via gold thermal evaporation, $10 \mathrm{CPA}$ nanoprobes.

The AFM morphological analysis of the CPA samples reveals that the replacement of the manual steps with controlled stages significantly improves the fabrication process, leading to a very high success rate, albeit evaluated on a limited number of samples. All the samples featured regular and well-defined patterns of nanospheres that are interrupted by only small and isolated defects. Specifically, to quantify this characteristic, we systematically analyzed the morphology of the 10 fibers by AFM scans over a $50 \times 50 \mu \mathrm{m}^{2}$ area in the center of the fiber tip (which includes the fiber core) and compared the results with similar samples obtained by the manual approach.

On the basis of the number of defects or the surface area covered by defects, the optimized process presents a clear improvement over the manual process demonstrated in our pioneering study. A detailed analysis of the AFM images is reported in Supplementary Fig. S2. Overall, the results reveal an average defective area of $0.5 \%$ by the optimized process, while a defective area of $1.5 \%$ was obtained (for the retained samples) by the manual process.

To analyze how the remaining defects affect the optical properties of the final structure, we recorded the transmittance spectra, displayed in Figure 7, which indicate good repeatability.

The spectra exhibit two repeatable resonances, as absorption dips, at 620 and $800 \mathrm{~nm}$. A similar transmittance spectrum was obtained in our previous work ${ }^{18}$ with a 1000 -nm CPA sample, but it exhibited only the 800-nm resonance, severely smoothed. The improvement in the sharpness of the plasmonic resonances can be attributed to the assessed fabrication process.

For the SA sample, we replicated the fiber probes with $1000-\mathrm{nm}$ MCCs five times, following the fabrication route described for the CPA nanoprobes. Then, the fibers were exposed to $400 \mathrm{~s}$ of plasma etching and were metallized to produce five SA fiber tips under the same conditions.

The SEM micrographs of the OFTs were processed using ImageJ software $^{34}$ to perform a grain analysis. Each image was adjusted by adjusting the 'Brightness/Contrast' and 'Window/Level' controls, and the threshold was regulated to produce binary images and a coherent mask of nanospheres.

To evaluate the homogeneity of the plasma size reduction on a single probe, we analyzed the same fiber in three spatial positions. The corresponding histograms showed reproducible diameter distributions, with an average value close to $828 \mathrm{~nm}$ (Supplementary Fig. S3) and a standard deviation of $4 \mathrm{~nm}$, corresponding to a coefficient of variation close to $0.5 \%$.

After the homogeneity within the sample was assessed, we evaluated the uniformity among the batch of samples in terms of the diameter and interparticle distance of the nanostructures. To this aim, the SEM images shown in Figure 8a were compared using the procedure described above. A summary of the obtained data is reported in Table 1 . As indicated by the distribution histograms in Supplementary Fig. S4, the particle diameter was uniformly reduced by the plasma treatment compared with the pristine CPA 1000-nm array. The average diameter was $834 \pm 9 \mathrm{~nm}$, whereas the average interparticle distance was $166 \pm 9 \mathrm{~nm}$. These results produced a coefficient of variation close to $1.1 \%$, which was similar to the coefficient of variation at the probe level.

To evaluate the effect that this level of repeatability has on the optical properties, we recorded the transmission spectra of the SA 

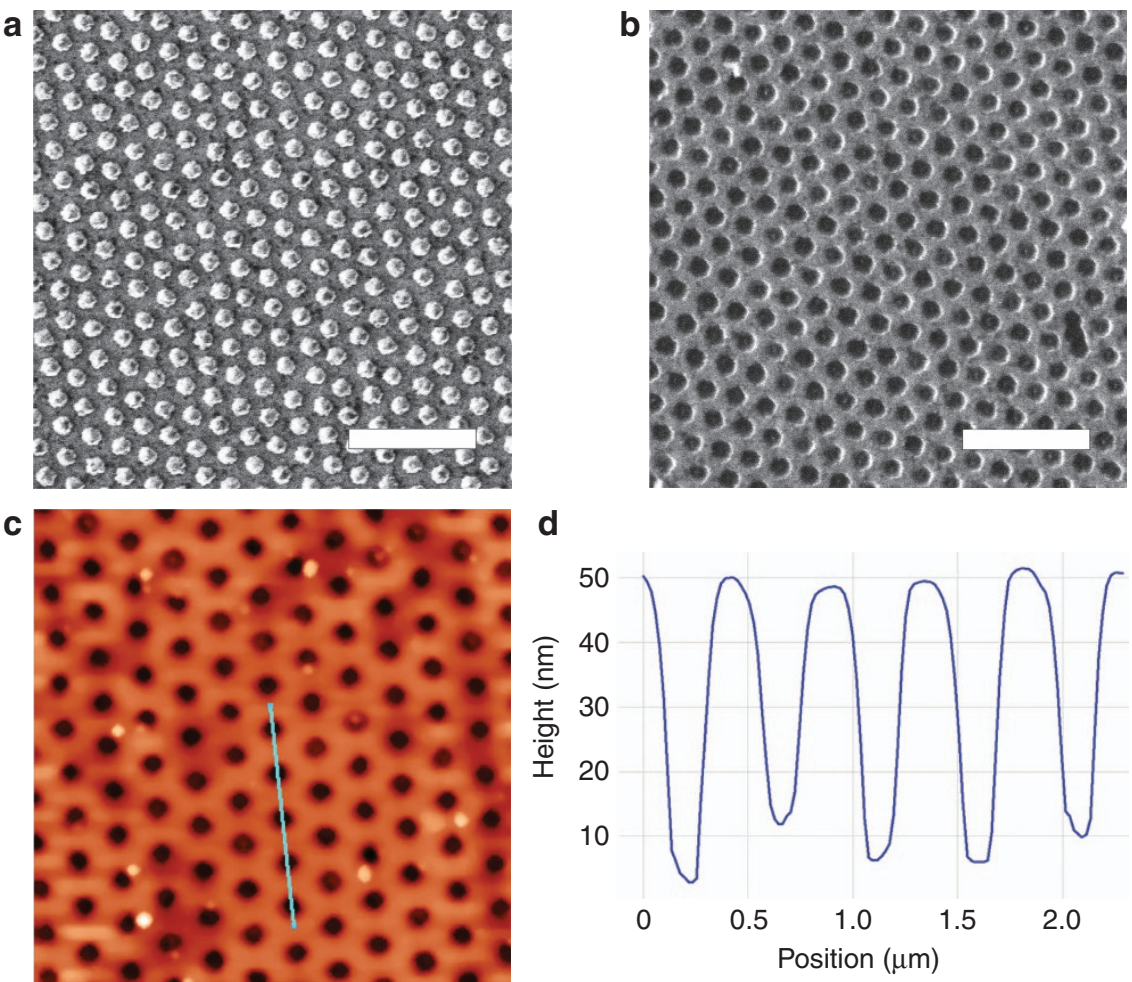

d
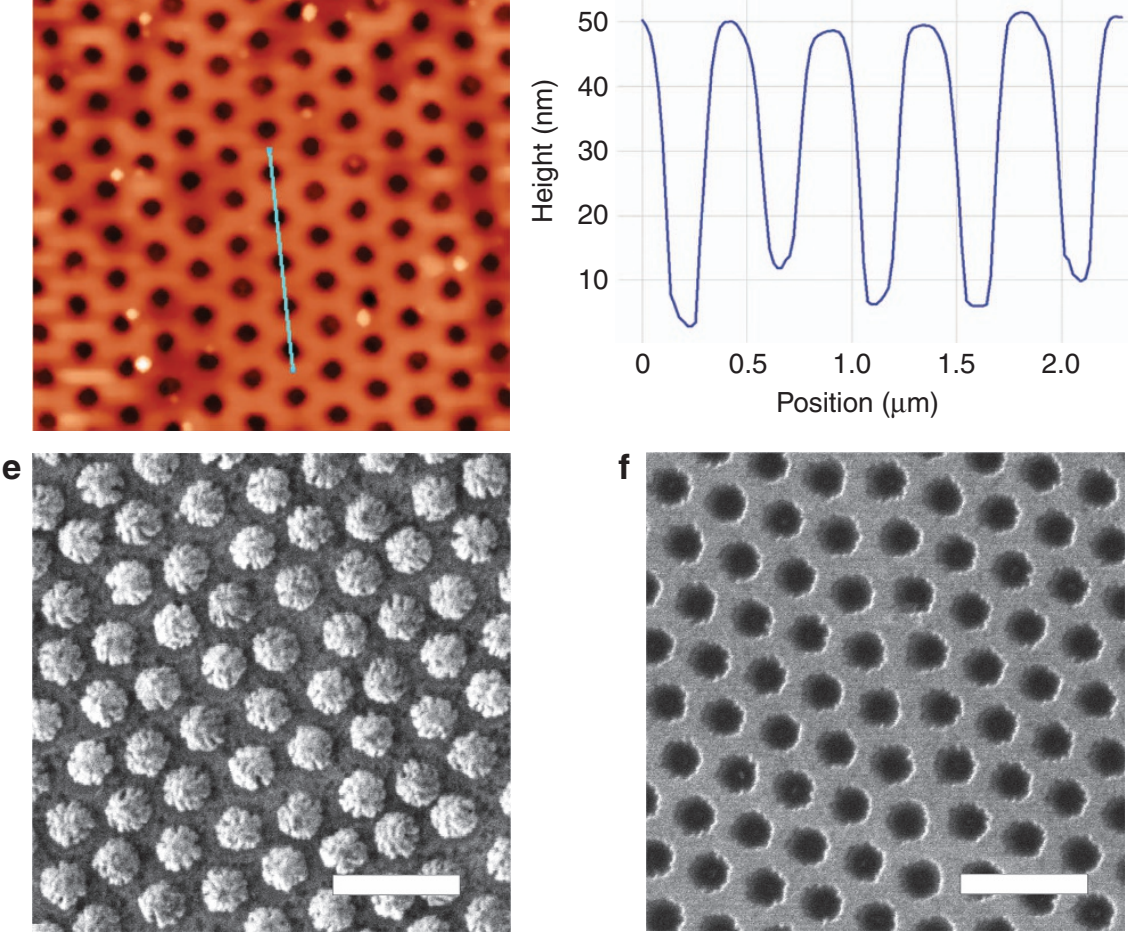

g

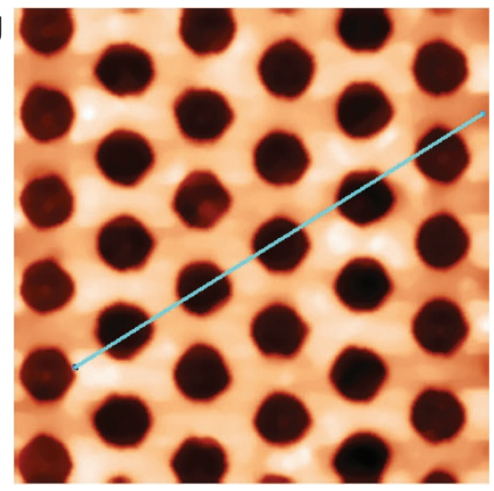

h

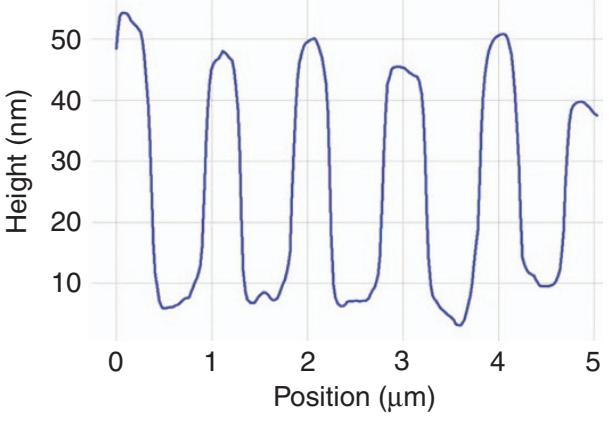

Figure 6 Fiber tips with SA patterns before (a, e) and after SR (b, c, f, g). The nanostructures were obtained starting with 500 (a-d) and $1000 \mathrm{~nm}$ (e-f) MCCs. (d, h) Section profiles measured along the light lines drawn in the AFM scans shown in $\mathbf{c}$ and $\mathbf{g}$, respectively. Scale bars $=3 \mu \mathrm{m}$.

nanoprobes, which are displayed in Figure 8b. The plots indicate satisfactory overlapping of the five curves, with two main adsorption dips at $\sim 650$ and $800 \mathrm{~nm}$. These results match the low coefficients of variation found by the morphological analysis, confirming the good reproducibility of the process steps.

\section{SERS measurements}

On the basis of the literature, we can envisage that the fabricated structures, if properly dimensioned, could be used for various sensing applications. Periodic metallic and metallo-dielectric patterns, if properly designed, can support surface plasmon resonance and/or 


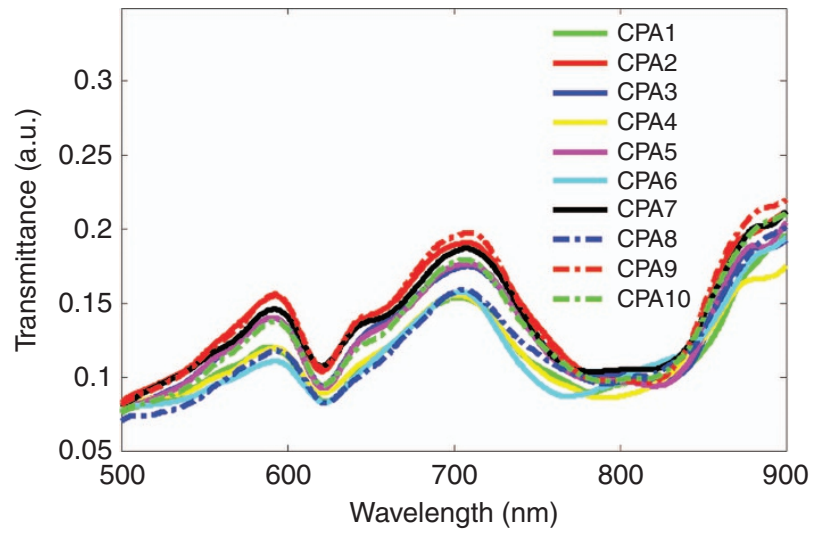

Figure 7 Transmittance spectra of the CPA nanoprobes produced with $1000 \mathrm{~nm}$ microspheres. localized surface plasmon resonance. When properly designed for specific wavelengths, these resonant features are spectrally evident and, in the electric field distribution, correspond to strong field localization at the boundaries of subwavelength-sized metallic structures. This superficial field enhancement yields resonant features that are strongly sensitive to local modifications in the external environment ${ }^{35,36}$, so they can be exploited for both chemosensing and biosensing applications. Similarly, SERS probes fabricated on OFTs potentially enable remote and in situ detection of molecules adsorbed onto the sensor surface ${ }^{16,37}$.

As a proof of concept, we demonstrate that our patterned tips can act as efficient SERS nanoprobes.

SERS efficiency is dependent on the plasmonic resonance of metallic nanostructures. Specifically, when the resonant wavelength of the nanostructure is between the wavelength of the Raman signal and the excitation wavelength, stronger SERS enhancement is
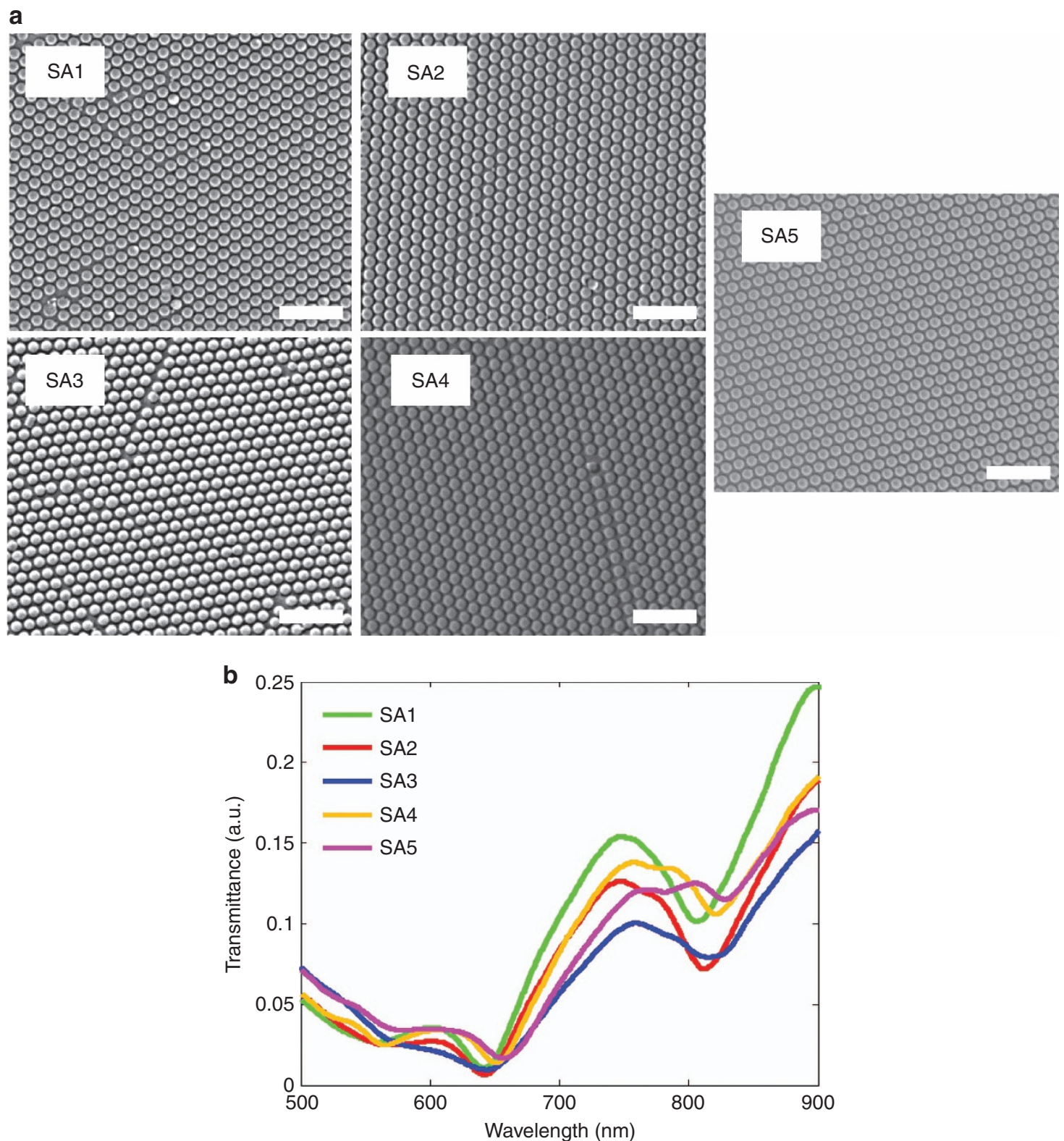

Figure 8 (a) SEM micrographs of five repeats of the SA nanoprobe prepared using 1000-nm PS spheres. The scale bars in the SEM images are $5 \mu \mathrm{m}$. (b) Overlapped transmission spectra of the five SA nanoprobes. 
Table 1 Grain analysis results on five SA nanoprobes

\begin{tabular}{lcc}
\hline & Average diameter $(\mathrm{nm})$ & Average interparticle closest distance $(\mathrm{nm})$ \\
\hline $\mathrm{SA} 1$ & 833 & 167 \\
$\mathrm{SA} 2$ & 823 & 178 \\
$\mathrm{SA3}$ & 828 & 172 \\
$\mathrm{SA} 4$ & 841 & 159 \\
SA5 & 846 & 154 \\
\hline
\end{tabular}

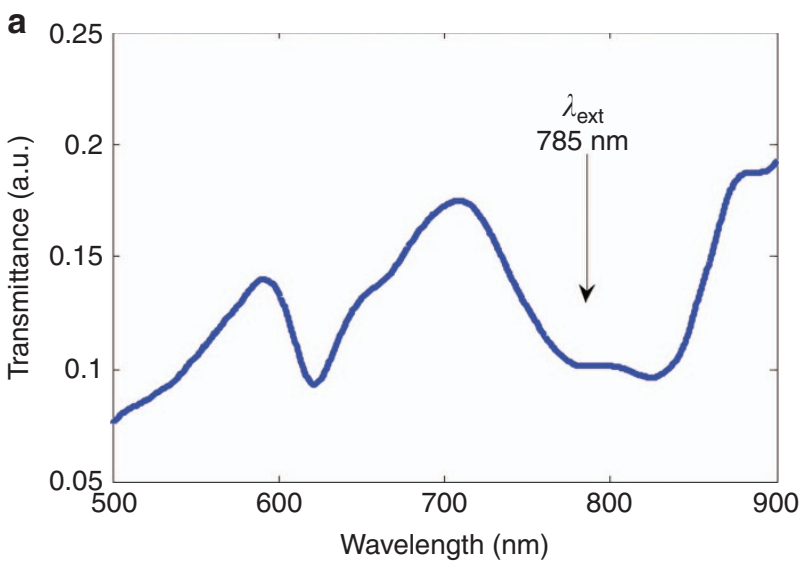

b

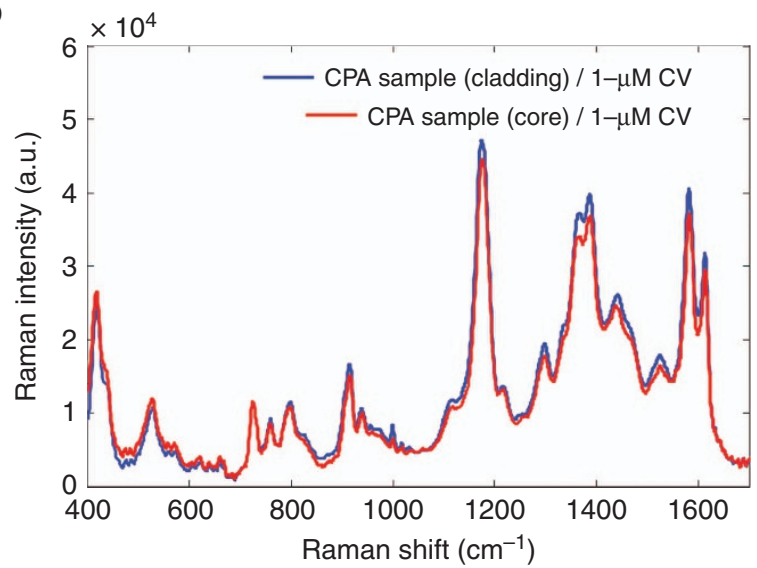

Figure 9 (a) Transmittance of the CPA sample with a sphere diameter of $1000 \mathrm{~nm}$. (b) SERS spectrum obtained from $1-\mu \mathrm{M}$ CV solution by illuminating the cladding (in blue) and core zones (in red) of the CPA sample.

obtained $^{38}$. Consequently, we selected one of the CPA samples (CPA3 in Figure 7) with a dip at $\sim 800 \mathrm{~nm}$ in the transmittance spectrum as a representative sample because this value is close to the excitation wavelength, that is, $785 \mathrm{~nm}$, of the Raman spectroscopy instrument that we used (Figure 9a).

As a benchmark, we used CV, a common dye, as a target molecule to test SERS substrates in both planar and fiber configurations ${ }^{16,39-42}$. The SERS measurements were performed following the procedure described in Yap et al. ${ }^{16}$. We prepared the SERS probes by soaking the fiber tip in $1-\mu \mathrm{M} \mathrm{CV}$ for $3 \mathrm{~h}$. Then, we focused the 785-nm excitation light on the CPA sample using a $50 \times$ objective lens. The scattered signal was collected by the same objective. The resulting SERS spectra related to the core and to the cladding regions of the tip are similar and are shown in Figure 9b, confirming the ordered and regular pattern over the fiber tip. In addition, the Raman signals from the CV vibrational modes at 915,1190, 1385 and $1617 \mathrm{~cm}^{-1}$ (Refs 43,44) are clear, despite the very low CV concentrations used in our experiment.

The detection performance overwhelms the results obtained in our preliminary report ${ }^{18}$. Despite using the same operating conditions, the Raman intensity doubled, and this performance improvement was enabled by the optimized fabrication process and the resulting sharp and well-defined plasmonic resonances.

Furthermore, the detection capability of our CPA probe is comparable to similar works related to SERS substrates using $\mathrm{CV}$ as a benchmark. In Table 2, we present some representative results ${ }^{16,40-43}$ for both the planar and fiber configurations, highlighting the structure under investigation, the fabrication process involved and the detected CV concentration.

A straight comparison can be made with Ref. 16, which used the same measurement methodology. The authors used a self-assembly process to obtain nanoparticle cluster arrays on the fiber tip and were able to detect $1-\mu \mathrm{M} \mathrm{CV}$.

Similarly, in the work of Liu et al. ${ }^{39}$, optical fiber SERS probes, composed of a porous polymer enriched with silver nanoparticles were fabricated at the terminal end of optical fibers. In this study, appreciable Raman intensity signals were observed for $0.1-\mu \mathrm{M} C V$, and clearly distinguishable peaks were observed only at higher concentrations (that is, $1 \mu \mathrm{M}$ of $\mathrm{CV}$ ).

Using SERS substrates in the planar configuration, Meng et al. ${ }^{40}$, with a SERS substrate featuring (disordered) silver colloids, detected concentrations as low as $0.1-\mu \mathrm{M} \mathrm{CV}$. Ouyang et al. ${ }^{41}$ synthesized a SERS substrate by fixing silver onto magnetite particles and detected 2.4- $\mu \mathrm{M} \mathrm{CV}$, whereas Bouvrée et al. used a planar substrate patterned and covered with $\mathrm{Au}$ nanoparticles to detect $1-\mu \mathrm{M} \mathrm{CV}^{42}$.

These results indicate that despite the advantages of using large planar substrates, the mentioned features are similar to those obtained in our case, where self-assembly is performed on the fiber tip.

The rigorous assessment of the probe performance for SERS applications requires a reliable estimation of the enhancement factor (EF) via estimation of the number of molecules contributing to the enhancement of the Raman signal ${ }^{45}$. Different experimental practices affect the molecule collection on the SERS substrate, so the detection capability, albeit a reasonable indicator of the SERS substrate quality, is not directly and quantitatively related to the EF. Specifically, in our measurements, careful quantification of the EF would require a reliable estimation of the surface density of the CV molecules on the samples, which is currently the subject of investigation.

Our effort in this study was not specifically focused on producing optimized SERS probes, but there is an optimization margin acting on the geometric features of the fabricated probes. In this stage, we mainly devoted our attention to the assessment and optimization of the fabrication process, which is a fundamental step to enable the realization of reproducible SERS substrates on OFTs, using a low-cost procedure. Nevertheless, these experimental results demonstrate that nanosphere self-assembly represents a valuable approach to produce regular nanostructures on OFTs, which could be exploited for various sensing applications.

\section{CONCLUSIONS}

We demonstrated that nanosphere lithography can be used to efficiently decorate OFTs with various periodic structures with submicrometric feature size and low fabrication cost. The proposed 
Table 2 Comparison of SERS substrates using CV as a benchmark

\begin{tabular}{|c|c|c|c|c|}
\hline Structure characteristics & Operating mode & Fabrication/preparation methods & $\begin{array}{l}\text { Target analyte (detection } \\
\text { concentration) }\end{array}$ & References \\
\hline $\begin{array}{l}\text { Porous polymer enriched with silver } \\
\text { nanoparticles }\end{array}$ & Fiber tip & $\begin{array}{l}\text { Thermal polymerization followed by photochemical } \\
\text { silver nanoparticle growth }\end{array}$ & $\mathrm{CV}\left(10^{-7} \mathrm{M}\right)$ & 39 \\
\hline Nanoparticle cluster array & Fiber tip & Block copolymer self-assembly & $\mathrm{CV}\left(10^{-6} \mathrm{M}\right)$ & 16 \\
\hline Uniform silver colloids & Planar substrate & Silver colloid reduction with sodium citrate & $\mathrm{CV}\left(10^{-7} \mathrm{M}\right)$ & 40 \\
\hline Silver/magnetite nanoparticles & Planar substrate & $\begin{array}{l}\mathrm{Fe}_{3} \mathrm{O}_{4} \text { : ultrasonic reverse co-precipitation method } \\
\mathrm{Ag}: \text { assisted chemical reaction }\end{array}$ & $\mathrm{CV}\left(2.4 \times 10^{-6} \mathrm{M}\right)$ & 41 \\
\hline $\begin{array}{l}\text { Nanostructured and nanopatterned } \\
\text { gold surfaces }\end{array}$ & Planar substrate & Electron beam lithography, AuNP solution soaking & $\mathrm{CV}\left(10^{-6} \mathrm{M}\right)$ & 42 \\
\hline
\end{tabular}

technique relies on the self-assembly of PS spheres at the AWI and their subsequent transfer to an OFT. The application of additional fabrication steps of thermal evaporation, plasma etching, and sonication generated four structural motifs (CPA, CPA-SR, SA and SA-SR), which were formed directly on the OFT and gave rise to four different metallo-dielectric periodic patterns. Plasma etching produced an anisotropic size reduction of the spheres, resulting in shape modifications that could be exploited to develop unexplored optical structures. This process worked with different sphere sizes within the dimensional limits of the optical fiber. AFM and FIBM morphological investigations of the OFTs established the efficacy of our nanopatterning procedure to form regular and well-ordered patterns. The reproducibility of the fabrication process was verified by the fabrication of different samples with the same nominal features. Morphological and spectral analyses confirmed the high reproducibility of the implemented fabrication steps. In addition, SERS measurements performed with one of the fabricated probes revealed that optical fibers patterned by this approach can be used for sensing applications. The detection capability of the SERS probe was comparable to similar works using both planar and fiber configurations with $\mathrm{CV}$ as a benchmark.

To the best of our knowledge, this study is the first report of a SERS signal observed using a periodically nanostructured fiber-probe surface patterned by a self-assembly process.

In conclusion, we believe that the straightforward nanofabrication approach proposed here is an easy method for patterning optical fibers with high precision at low cost and has potential applications in the field of 'lab-on-fiber' technology.

\section{CONFLICT OF INTEREST}

The authors declare no conflict of interest.

1 Kostovski G, Stoddart PR, Mitchell A. The optical fiber tip: an inherently light-coupled microscopic platform for micro- and nanotechnologies. Adv Mater 2014; 26: 3798-3820.

2 Pisco M, Quero G, ladicicco A, Giordano M, Galeotti F et al. Lab on fiber by using the breath figure technique. In: Cusano A, Consales M, Crescitelli A, Ricciardi A, editors. Lab-on-Fiber Technology. Switzerland: Springer International Publishing; 2015, p233-250.

3 Ricciardi A, Crescitelli A, Vaiano P, Quero G, Consales M et al. Lab-on-fiber technology: a new vision for chemical and biological sensing. Analyst 2015; 140: 8068-8079.

4 Consales M, Ricciardi A, Crescitelli A, Esposito E, Cutolo A et al. Lab-on-fiber technology: toward multifunctional optical nanoprobes. ACS Nano 2012; 6: 3163-3170.

5 Lin YB, Zou Y, Lindquist RG. A reflection-based localized surface plasmon resonance fiber-optic probe for biochemical sensing. Biomed Opt Express 2011; 2: 478-484.

6 Sanders M, Lin YB, Wei JJ, Bono T, Lindquist RG. An enhanced LSPR fiber-optic nanoprobe for ultrasensitive detection of protein biomarkers. Biosens Bioelectron 2014; 61: $95-101$
7 Micco A, Ricciardi A, Pisco M, La Ferrara V, Cusano A. Optical fiber tip templating using direct focused ion beam milling. Sci Rep 2015; 5: 15935.

8 Dhawan A, Gerhold M, Madison A, Fowlkes J, Russell PE et al. Fabrication of nanodot plasmonic waveguide structures using FIB milling and electron beam-induced deposition. Scanning 2009; 31: 139-146.

9 Dhawan A, Gerhold MD, Muth JF. Plasmonic structures based on subwavelength apertures for chemical and biological sensing applications. IEEE Sens J 2008; 8: 942-950.

$10 \mathrm{Jia}$ PP, Yang J. Integration of large-area metallic nanohole arrays with multimode optical fibers for surface plasmon resonance sensing. App/ Phys Lett 2013; 102: 243107.

11 Whitesides GM, Grzybowski B. Self-assembly at all scales. Science 2002; 295 : 2418-2421.

12 Mullen KI, Carron KT. Surface-enhanced Raman spectroscopy with abrasively modified fiber optic probes. Anal Chem 1991; 63: 2196-2199.

13 Andrade GFS, Fan MK, Brolo AG. Multilayer silver nanoparticles-modified optical fiber tip for high performance SERS remote sensing. Biosens Bioelectron 2010; 25 : 2270-2275.

14 Viets C, Hill W. Comparison of fibre-optic SERS sensors with differently prepared tips. Sens Actuat B Chem 1998; 51: 92-99.

15 Stoddart PR, White PA, Mazzolini A. Chemical sensors based on nanoparticle arrays. Proc SPIE 2002; 4934: 61-69.

16 Yap FL, Thoniyot P, Krishnan S, Krishnamoorthy S. Nanoparticle cluster arrays for highperformance SERS through directed self-assembly on flat substrates and on optical fibers. ACS Nano 2012; 6: 2056-2070.

17 Pisco M, Galeotti F, Quero G, ladicicco A, Giordano M et al. Miniaturized sensing probes based on metallic dielectric crystals self-assembled on optical fiber tips. ACS Photonics 2014; 1: 917-927.

18 Pisco M, Galeotti F, Quero G, Grisci G, Micco A et al. Nanosphere lithography for advanced all fiber Sers probes. Proc SPIE 2016; 9916: 99161S.

19 Ye XZ, Qi LM. Two-dimensionally patterned nanostructures based on monolayer colloidal crystals: controllable fabrication, assembly, and applications. Nano Today 2011; 6: 608-631.

20 Moon GD, Lee TI, Kim B, Chae G, Kim J et al. Assembled monolayers of hydrophilic particles on water surfaces. ACS Nano 2011; 5: 8600-8612.

21 Ho CC, Chen PY, Lin KH, Juan WT, Lee WL. Fabrication of monolayer of polymer/ nanospheres hybrid at a water-air interface. ACS Appl Mater Interfaces 2011; 3: 204-208.

22 Yu J, Yan QF, Shen DZ. Co-self-assembly of binary colloidal crystals at the air-water interface. ACS App/ Mater Interfaces 2010; 2: 1922-1926.

23 Dai ZF, Li Y, Duan GT, Jia LC, Cai WP. Phase diagram, design of monolayer binary colloidal crystals, and their fabrication based on ethanol-assisted self-assembly at the air/water interface. ACS Nano 2012; 6: 6706-6716.

24 Kitaev V, Ozin GA. Self-assembled surface patterns of binary colloidal crystals. Adv Mater 2003; 15: 75-78.

25 Wang LK, Wan Y, Li YQ, Cai ZY, Li HL et al. Binary colloidal crystals fabricated with a horizontal deposition method. Langmuir 2009; 25: 6753-6759.

26 Zhou ZC, Yan QF, Li Q, Zhao XS. Fabrication of binary colloidal crystals and non-closepacked structures by a sequential self-assembly method. Langmuir 2007; 23: 1473-1477.

27 Haynes CL, van Duyne RP. Nanosphere lithography: a versatile nanofabrication tool for studies of size-dependent nanoparticle optics. J Phys Chem B 2001; 105: 5599-5611.

28 Pisco M, Galeotti F, Grisci G, Quero G, Cusano A. Self-assembled periodic patterns on the optical fiber tip by microsphere arrays. Proc SPIE 2015; 9634: 96341N.

29 Muldarisnur M, Marlow F. Opal films made by the capillary deposition method: crystal orientation and defects. J Phys Chem C 2011; 115: 414-418.

30 Hoogenboom JP, Derks D, Vergeer $P$, van Blaaderen A. Stacking faults in colloidal crystals grown by sedimentation. J Chem Phys 2002; 117: 11320-11328.

31 Jiang P, McFarland MJ. Large-scale fabrication of wafer-size colloidal crystals, macroporous polymers and nanocomposites by spin-coating. J Am Chem Soc 2004; 126: $13778-13786$ 
32 Ariga K, Yamauchi Y, Mori T, Hill JP. 25th anniversary article: what can be done with the langmuir-blodgett method? Recent developments and its critical role in materials science. Adv Mater 2013; 25: 6477-6512.

33 Akinoglu EM, Morfa AJ, Giersig M. Understanding anisotropic plasma etching of two-dimensional polystyrene opals for advanced materials fabrication. Langmuir 2014; 30: $12354-12361$.

34 Schneider CA, Rasband WS, Eliceiri KW. NIH Image to Image J: 25 years of image analysis. Nat Methods 2012; 9: 671-675.

35 Homola J. Surface plasmon resonance sensors for detection of chemical and biological species. Chem Rev 2008; 108: 462-493.

36 Stewart ME, Anderton CR, Thompson LB, Maria J, Gray SK et al. Nanostructured plasmonic sensors. Chem Rev 2008; 108: 494-521.

37 Smythe EJ, Dickey MD, Bao JM, Whitesides GM, Capasso F. Optical antenna arrays on a fiber facet for in situ surface-enhanced Raman scattering detection. Nano Lett 2009; 9: 1132-1138.

38 Kahraman M, Daggumati P, Kurtulus O, Seker E, Wachsmann-Hogiu S. Fabrication and characterization of flexible and tunable plasmonic nanostructures. Sci Rep 2013; 3: 3396.

39 Liu CY, Wang SY, Chen G, Xu SP, Jia Q et al. A surface-enhanced Raman scattering (SERS)-active optical fiber sensor based on a three-dimensional sensing layer. Sens Biosensing Res 2014; 1: 8-14.

40 Meng W, Hu F, Jiang XH, Lu LD. Preparation of silver colloids with improved uniformity and stable surface-enhanced Raman scattering. Nanoscale Res Lett 2015; 10: 34.
41 Ouyang L, Zhu LH, Jiang JZ, Tang HQ. A surface-enhanced Raman scattering method for detection of trace glutathione on the basis of immobilized silver nanoparticles and crystal violet probe. Anal Chim Acta 2014; 816: 41-49.

42 Bouvrée A, D'Orlando A, Makiabadi T, Martin S, Louarn G et al. Nanostructured and nanopatterned gold surfaces: application to the surface-enhanced Raman spectroscopy. Gold Bull 2013; 46: 283-290.

43 Kudelski A. Raman studies of rhodamine $6 \mathrm{G}$ and crystal violet sub-monolayers on electrochemically roughened silver substrates: do dye molecules adsorb preferentially on highly SERS-active sites? Chem Phys Lett 2005; 414: 271-275.

44 Angeloni L, Smulevich G, Marzocchi MP. Resonance Raman spectrum of crystal violet. J Raman Spectrosc 1979; 8: 305-310.

45 Le Ru EC, Etchegoin PG. Quantifying SERS enhancements. MRS Bull 2013; 38: 631-640.

cc)(1) (2) This work is licensed under a Creative Commons AttributionNonCommercial-ShareAlike 4.0 International License. The images or other third party material in this article are included in the article's Creative Commons license, unless indicated otherwise in the credit line; if the material is not included under the Creative Commons license, users will need to obtain permission from the license

holder to reproduce the material. To view a copy of this license, visit http:// creativecommons.org/licenses/by-nc-sa/4.0/

(C) The Author(s) 2017

Supplementary Information for this article can be found on the Light: Science \& Applications' website (http://www.nature.com/lsa). 